\title{
The Role of Mean Platelet Volume in Chronic Obstructive Pulmonary Disease Exacerbation
}

\author{
Dimitris I Agapakis MD, Eleni V Massa MD MSc, Ioannis Hantzis MD, Stefanos Maraslis MD, \\ Evagelos Alexiou MD, Konstantinos P Imprialos MD, Maria Damianidou MD, and \\ Emilios Satsoglou MD
}

\begin{abstract}
BACKGROUND: Studies have supported the correlation between mean platelet volume and COPD. However, there are limited data on the relationship between COPD exacerbation and mean platelet volume. We aimed to evaluate the mean platelet volume trend in patients with COPD exacerbation. METHODS: A total of 81 subjects, 62 men and 19 women, who were admitted to the hospital because of exacerbation of COPD during 9 months, were enrolled in this prospective observational study. The levels of mean platelet volume, $C$-reactive protein, complete blood count, and percentof-predicted $\mathrm{FEV}_{1}$ were measured in subjects at admission (exacerbation period) and after 3 months (stable period). Thirty-seven age- and sex-matched healthy individuals constituted the control group. RESULTS: Subjects in the exacerbation period had significantly higher levels of C-reactive protein $(P=.001)$, white blood cell count $(P=.01)$, and percentage of neutrophils $(P=.01)$ and lower percent-of-predicted $F E V_{1}$ than in the stable period $(P=.02)$. Mean platelet volume levels were significantly decreased in the exacerbation period $(P=.001)$. Considering a cut-off point of mean platelet volume levels $<8.2 \mathrm{fL}$ for indicating COPD exacerbation showed a sensitivity of $80 \%$ and a specificity of $76 \%$. Also, mean platelet volume levels correlated significantly with increase of $\mathrm{C}$-reactive protein level, white blood cell count, and neutrophil percentage in the exacerbation period $(P=.01, P=.01$, and $P=.02$, respectively). CONCLUSIONS: Mean platelet volume may be an inflammatory marker in exacerbation of COPD, and the measurement of mean platelet volume values may be useful for identifying patients who are at increased risk for exacerbations of illness. Key words: mean platelet volume; exacerbation; COPD; inflammation; prediction; discrimination. [Respir Care 2016;61(1):44-49. (C) 2016 Daedalus Enterprises]
\end{abstract}

\section{Introduction}

COPD is a major global health problem with an upward trend. It is characterized by progressive air flow limitation

\footnotetext{
Drs Agapakis, Hantzis, Maraslis, Imprialos, and Satsoglou are affiliated with the Department of Internal Medicine, General Hospital of Goumenissa, Goumenissa, Greece. Dr Alexiou is affiliated with the Department of Radiology, General Hospital of Goumenissa. Dr Damianidou is affiliated with the Department of Microbiology, General Hospital of Goumenissa. Dr Massa is affiliated with the ICU, General Hospital Hippokratio, Thessaloniki, Greece.
}

The authors have disclosed no conflicts of interest.

Correspondence: Dimitris I Agapakis MD. E-mail: dimagap@yahoo.gr.

DOI: $10.4187 /$ respcare.04132 that is not fully reversible and is associated with an abnormal inflammatory response of the lung to noxious particles or gases. ${ }^{1}$ On the other hand, exacerbations occur commonly in patients with COPD; contribute to disease severity; and are implicated in the quality of life, morbidity, and mortality of these patients. ${ }^{2}$ Therefore, a strategy for prevention and early treatment of COPD exacerbations is essential to better address the disease. However, its assessment is based primarily on clinical presentations, which can be variable, which complicates their interpretation. Therefore, several biomarkers have already been assessed in this context without wide acceptance. ${ }^{3}$

Also, it is known that platelets play a major role in inflammation as in thrombosis. ${ }^{4}$ Mean platelet volume is a measure of platelet size and is correlated in general with their activity. ${ }^{5}$ Mean platelet volume disturbances reflect changes in either the level of platelet stimulation or the 
rate of platelet production. ${ }^{6}$ Moreover, mean platelet volume has been used as an indicator of platelet function for inflammatory diseases, ${ }^{7}$ and because inflammation plays a crucial role in COPD, alterations in platelet activity and consequently in the mean platelet volume are expected. Indeed, there are various studies that show a disturbance of mean platelet volume levels in subjects with stable COPD. ${ }^{8,9}$

However, the association of mean platelet volume with COPD exacerbation has not yet been adequately evaluated. In recent years, there have been only limited studies evaluating this issue with controversial results. Some studies have shown that mean platelet volume levels are lower during exacerbation compared with the stable phase of COPD,${ }^{10,11}$ whereas a report found that this association is not statistically significant. ${ }^{12}$ The aim of the present study was to investigate the relationship between exacerbations and mean platelet volume trend in subjects with COPD and to clarify its significance as an inflammatory biomarker in COPD exacerbation.

\section{Methods}

\section{Study Design}

We studied 81 subjects who were hospitalized with a diagnosis of COPD exacerbation in the Department of Internal Medicine of our hospital for a duration of 9 months. All were receiving regular inhaled long-acting $\beta_{2}$-agonists, regular inhaled glucocorticoids, and inhaled tiotropium bromide. COPD exacerbation was diagnosed by the presence of sustained (lasting $\geq 48 \mathrm{~h}$ ) increased breathlessness or dyspnea; increased cough or sputum production; or a sputum color change that was beyond normal day-to-day variations, was acute in onset, and led to an increase in the use of maintenance medications and/or supplementation with additional medications for patients with COPD, according to Global Initiative for Chronic Obstructive Lung Disease guidelines. ${ }^{1}$ Also, COPD was diagnosed according to the above guidelines based on past smoking history, clinical evaluation, and pulmonary function tests showing irreversible air flow obstruction. ${ }^{1}$

We excluded from the study patients with coronary artery disease; acute heart failure; pneumonia; pulmonary thromboembolism; diabetes mellitus; or a history of cystic fibrosis, coagulation disorders, malignancy, connective tissue disease, or inflammatory bowel disease that could affect the mean platelet volume. Also, patients with prior treatment with anticoagulant regimens or anti-platelet medications were excluded. The institutional review board of the hospital approved the research protocol, which complied with the 2000 Declaration of Helsinki, and all subjects consented to participate in the study.

\section{QUICK LOOK}

\section{Current knowledge}

COPD is a chronic disease characterized by progressive air flow limitation for which there is no cure. The global burden of COPD is significant and growing. Chronic inflammation, recurrent infections, and hospitalizations for exacerbations are common findings in advanced COPD.

\section{What this paper contributes to our knowledge}

Mean platelet volume was significantly reduced during a COPD exacerbation. Mean platelet volume closely tracked increases in C-reactive protein. The role of mean platelet volume as an inflammatory marker in COPD exacerbations or for identifying patients at increased risk for exacerbations of illness remains to be determined.

\section{Clinical and Laboratory Investigations}

Clinical data were recorded, and blood samples were obtained from each subject at admission to the hospital before starting treatment. Complete blood counts and Creactive protein levels were measured in peripheral blood (exacerbation period). Also, spirometry was performed, and percent-of-predicted $\mathrm{FEV}_{1}$ was measured. Thirty-seven healthy age- and sex-matched individuals were included in the study as a control group. Subjects were seen in convalescence, when clinically stable, 3 months later, and all clinical assessments and sampling were repeated (stable period).

Hematological parameters were determined on a hematology analyzer (Beckman Coulter, Brea, California), which was standardized for mean platelet volume measurement, whereas the CRPLX Tina-quant kit (Roche-Hitachi, Basel, Switzerland) was used for C-reactive protein concentration measurement. A MicroLab 3500 spirometer (CareFusion, San Diego, California) was used for lung function evaluation. Potential confounders in this study were age, sex, smoking, and comorbidities.

\section{Statistical Analysis}

Data are reported as means $\pm \mathrm{SD}$. The chi-square test was used for categorical variables. The Student $t$ test was performed to compare the parameters between COPD exacerbation and controls, and a paired $t$ test was used to compare the parameters between COPD exacerbation and stable COPD. Pearson correlation analysis was used to explore the correlation between parameters. The diagnostic usefulness of serum mean platelet volume value was assessed by means of the receiver operating characteristic curve, according to sensitivity and specificity for different 
Table 1. Baseline Characteristics of the 81 Subjects

\begin{tabular}{lccc}
\hline \hline \multicolumn{1}{c}{ Characteristic } & $\begin{array}{c}\text { COPD } \\
\text { Exacerbation }\end{array}$ & Control & $P$ \\
\hline Age, mean \pm SD y & $65.3 \pm 11.1$ & $62.19 \pm 12.7$ & .45 \\
Male sex, \% & 78.7 & 72.4 & .36 \\
Smokers, \% & 22.9 & 20.7 & .63 \\
WBC count, mean \pm SD $\times 10^{3} / \mu 1$ & $10.53 \pm 3.48$ & $7.43 \pm 1.58$ & $<.01$ \\
Neutrophils, mean \pm SD \% & $78 \pm 9.8$ & $65 \pm 13.1$ & $<.01$ \\
Hct, mean \pm SD \% & $43 \pm 1.1$ & $42 \pm 0.7$ & .71 \\
PLT, mean \pm SD $\times 10^{3} / \mu 1$ & $243 \pm 55$ & $219 \pm 95$ & $<.05$ \\
CRP, mean \pm SD mg/dl & $3.9 \pm 2.3$ & $0.5 \pm 1.1$ & $<.001$ \\
MPV, mean \pm SD fL & $8.5 \pm 0.9$ & $9.1 \pm 0.9$ & .001 \\
FEV ${ }_{1}$, mean \pm SD \% predicted & $47.5 \pm 15.9$ & $121.1 \pm 11.3$ & .001 \\
& & & \\
\hline WBC $=$ white blood cell & & & \\
Hct $=$ hematocrit & & & \\
PLT $=$ platelet & & & \\
CRP $=$ C-reactive protein & & & \\
MPV $=$ mean platelet volume & & & \\
&
\end{tabular}

cut-off points, for discriminating subjects with COPD exacerbation from those with stable COPD. For the optimum cut-off point provided by receiver operating characteristic analysis, sensitivity, specificity, positive predictive value, and negative predictive value were calculated using a standard formula. The relative risk for having COPD exacerbation was estimated by the odds ratio and $95 \%$ CI. $P<.05$ was considered significant. Statistical analyses were conducted with SPSS 20 (SPSS, Chicago, Illinois).

\section{Results}

Characteristics of subjects and controls are summarized in Table 1. The mean age of the subjects was $65.3 \pm 11.1 \mathrm{y}$, $62(76.5 \%)$ were men, and $18(22.2 \%)$ were current smokers. Subjects and controls did not differ in age, sex, smoking status, and hematocrit and hemoglobin levels. The platelet count, the WBC count, the neutrophil percentage, and C-reactive protein levels were significantly higher $(P<.05$, $P<.01, P<.01$, and $P<.001$, respectively), whereas mean platelet volume and percent-of-predicted $\mathrm{FEV}_{1}$ were significantly lower in subjects with COPD exacerbation compared with controls $(P=.001$ and $P=.001$, respectively). These differences persisted when study subjects were stratified by age group and sex.

The comparison of measured parameters in exacerbation and stable COPD is shown in Table 2. Subjects in the COPD exacerbation period had significantly higher levels of C-reactive protein, WBC count, and neutophil percentage than in the period of stable disease $(P=.001, P=.01$, and $P=.01$, respectively). The platelet count was higher in COPD exacerbation, but the difference with stable COPD did not reach significance $(P=.08)$. The mean platelet volume levels were significantly lower in the exacerbation
Table 2. Comparison of Measured Parameters of Subjects in Exacerbation Phase and Stabilization Phase of COPD

\begin{tabular}{lccc}
\hline \hline & COPD Exacerbation & Stable COPD & $P$ \\
\hline $\mathrm{MPV}, \mathrm{fL}$ & $8.5 \pm 0.9$ & $9.3 \pm 1.3$ & .001 \\
$\mathrm{CRP}, \mathrm{mg} / \mathrm{dl}$ & $3.9 \pm 2.3$ & $2.1 \pm 0.7$ & .001 \\
$\mathrm{PLT}, \times 10^{3} / \mu \mathrm{l}$ & $243 \pm 55$ & $227 \pm 73$ & .08 \\
$\mathrm{WBC}, \times 10^{3} / \mu \mathrm{l}$ & $10.53 \pm 3.48$ & $8.47 \pm 2.78$ & .01 \\
Neutrophils $\%$ & $78 \pm 9.8$ & $71 \pm 5.7$ & .01 \\
$\mathrm{FEV}_{1}, \%$ predicted & $47.5 \pm 15.9$ & $54.7 \pm 17.7$ & .02 \\
$\mathrm{P}_{\mathrm{aO}}, \mathrm{mm} \mathrm{Hg}$ & $57 \pm 5.2$ & $60 \pm 4.7$ & .02 \\
& & & \\
\hline Data are presented as means $\pm \mathrm{SD}$. & & & \\
$\mathrm{MPV}=$ mean platelet volume & & & \\
$\mathrm{CRP}=\mathrm{C}$-reactive protein & & & \\
$\mathrm{PLT}=$ platelet & & & \\
$\mathrm{WBC}=$ white blood cell & & & \\
& & & \\
\hline
\end{tabular}

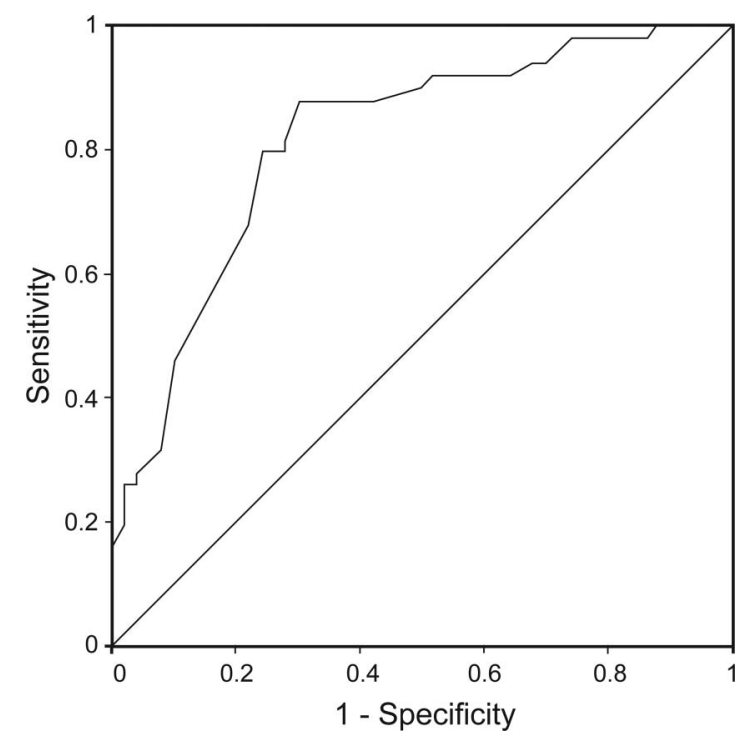

Fig. 1. A receiver operating characteristic curve is shown with the best cut point of mean platelet volume ( $8.2 \mathrm{fL})$, which is suggested to discriminate between the acute exacerbation phase and the stabilization phase of COPD at the time of diagnosis (sensitivity of $80 \%$, specificity of $76 \%$; area under the curve, 0.818 ; SE $=0.043$; $P<.001 ; 95 \% \mathrm{Cl} 0.735-0.902)$.

period compared with stable COPD $(P=.001)$. Moreover, a cut-off point of $8.2 \mathrm{fL}$ would discriminate between exacerbation and stable COPD with a sensitivity of $80 \%$ and a specificity of $76 \%$ and could be proposed as an indicative parameter for COPD exacerbation appearance $(95 \% \mathrm{CI}$ 0.735-0.902; area under the receiver operating characteristic curve, 0.818) (Fig. 1). The negative predictive value and positive predictive value for this cut-off point of mean platelet volume level were 76 and $78 \%$, respectively. Also, mean percent-of-predicted $\mathrm{FEV}_{1}$ was significantly lower in the exacerbation period $(P=.02)$. In contrast, there were no significant differences regarding hematocrit and hemoglobin levels in the two periods $(P>.05$ for all 
Table 3. Correlation of Mean Platelet Volume With C-Reactive Protein Level, White Blood Cell Count, Neutrophil Percentage, Percent-ofPredicted $\mathrm{FEV}_{1}$, and Duration of Hospital Stay in Exacerbation Period

\begin{tabular}{|c|c|c|c|c|}
\hline CRP & WBC & Neutrophils & Percent-of-Predicted $\mathrm{FEV}_{1}$ & Hospital Stay \\
\hline $\mathrm{r}=-0.4, P=.01$ & $\mathrm{r}=-0.5, P=.01$ & $\mathrm{r}=-0.4, P=.02$ & $\mathrm{r}=0.04, P=.34$ & $\mathrm{r}=0.05, P=.72$ \\
\hline $\begin{array}{l}\mathrm{CRP}=\mathrm{C} \text {-reactive protein } \\
\mathrm{WBC}=\text { white blood cell } \\
\mathrm{MPV}=\text { mean platelet volume }\end{array}$ & & & & \\
\hline
\end{tabular}

comparisons). The mean time of hospital stay was $4.3 \pm 1.9$ d (range $3-8 \mathrm{~d}$ ). No subjects died.

Mean platelet volume was significantly correlated with C-reactive protein, WBC count, and neutrophil percentage in the exacerbation period $(\mathrm{r}=-0.4, P=.01 ; \mathrm{r}=-0.5$, $P=.01$; and $\mathrm{r}=-0.45, P=.02$, respectively). In contrast, no significant correlation between mean platelet volume and $\mathrm{FEV}_{1}$ was found $(\mathrm{r}=0.04, P=.34)$. Also, there was no correlation between mean platelet volume and duration of hospitalization $(P=.72)$. Data are shown in Table 3.

\section{Discussion}

This observational study reveals that the mean platelet volume levels in subjects with COPD exacerbation decreased compared with the stable period and with healthy individuals. Also, the mean platelet volume levels were correlated with an increased C-reactive protein and increases in both the relative proportion of neutrophils and the WBC count in subjects with COPD exacerbation.

Consequently, the present study suggests that mean platelet volume levels may be a helpful inflammatory biomarker indicating COPD exacerbation. Especially, levels of mean platelet volume $<8.2 \mathrm{fL}$ may discriminate the stable from the exacerbation period of COPD.

With regard to the changes in mean platelet volume levels, our findings are in line with the results of a study by Ulasli et $\mathrm{al}^{10}{ }^{10}$ who demonstrated that in the exacerbation period of COPD, mean platelet volume levels were decreased. On the contrary, they failed to detect a correlation between mean platelet volume and C-reactive protein, probably due to their small sample size, the baseline increased level of C-reactive protein, or the high mean platelet volume levels in the stable period. Similarly, in a recent study, Wang et al ${ }^{11}$ reported that mean platelet volume values were lower during the exacerbation phase compared with the stable phase of COPD. Also, WBC count and C-reactive protein were positively related with reduced mean platelet volume as we found. However, the above studies did not determine any threshold mean platelet volume level indicating the exacerbation phase as we did. A mean platelet volume level of $8.2 \mathrm{fL}$ would dis- criminate between the two phases of COPD with a sensitivity of $80 \%$ and a specificity of $76 \%$.

But why are mean platelet volume levels decreased in COPD exacerbation? The literature does not support an accurate mechanism to fully explain these results, so we'll try to formulate some explanations.

First of all, since the inflammation is a core feature of COPD exacerbation, it could be the cause of such a change in level of mean platelet volume. This present inflammatory response, caused mostly by bacterial infections of lower airways, is higher than in the stable disease. ${ }^{13}$ Potential mechanisms for this increased systemic inflammation in COPD exacerbation include: (1) spillover of inflammatory mediators from the pulmonary compartment; (2) an inflammatory reaction to tissue hypoxia; and (3) a reaction induced by the pro-inflammatory bacterial product lipopolysaccharide. ${ }^{14}$ Several studies showed that inflammatory markers, such as circulating neutrophil numbers, C-reactive protein, fibrinogen, interleukin-6, interleukin-8, and soluble intercellular adhesion molecule 1, are increased during COPD exacerbation. ${ }^{15,16}$ In our study, WBC count and the percentage of neutrophils were significantly higher compared with during the stable period, as were the C-reactive protein levels, although not normalized, as expected. ${ }^{16}$

The investigators of the above studies ${ }^{10,11}$ favor this view. However, the intensity of inflammation during COPD exacerbation could lead to reverse results (ie, increased mean platelet volume). Furthermore, increased levels of proinflammatory cytokines and C-reactive protein can lead to the production of more reactive large platelets. ${ }^{17,18}$ For example, a small study by Erden et al $^{12}$ showed that mean platelet volume levels were increased in COPD exacerbation subjects, although the difference did not reach significance.

At the same time, COPD exacerbation has been associated with a prothrombotic state. Elevated levels of von Willebrand factor, D-dimer, and prothrombin fragment $1+2$, being surrogate markers for inflammation, endothelial damage, and clotting activation, respectively, have been measured during an exacerbation of COPD. ${ }^{19} \mathrm{~A}$ number of studies have demonstrated that subjects with COPD have an increased platelet count and also increased platelet ac- 


\section{Mean Platelet Volume in COPD Exacerbation}

tivation in stable disease and further in the exacerbation period. ${ }^{8,20}$

However, this platelet activation can follow different patterns as to mean platelet volume values in various diseases. In particular, in diseases with low-grade chronic inflammation that predisposes to thrombosis, such as cardiovascular disease or atherosclerosis (tendency to hypercoagulability), mean platelet volume levels are increased. In contrast, in diseases with high-grade inflammation, such as active rheumatoid arthritis, inflammatory bowel disease, and familial Mediterranean fever, mean platelet volume levels are reduced and normalized by anti-inflammatory therapy. ${ }^{21}$ In our study, COPD exacerbation was associated with a significant decrease of mean platelet volume levels, such as in the high-grade inflammation diseases. However, because the great majority of severe COPD exacerbations requiring hospitalization are infectious in etiology,22 a key difference from the previous inflammatory findings is that in the exacerbation period, an infection is present. It could be argued that infections of airways may trigger abnormalities in inflammation and thrombosis markers, such as mean platelet volume, a link between inflammation and thrombosis. ${ }^{23}$ Along these lines, it has been found that children with cystic fibrosis have decreased levels of mean platelet volume during the exacerbation period compared with the stable phase. ${ }^{24} \mathrm{Sim}-$ ilarly, mean platelet volume levels in subjects with asthma with exacerbations were lower compared with those in subjects with stable asthma. ${ }^{25}$ Finally, the possibility cannot be excluded that the reduction in mean platelet volume is due to the consumption or sequestration of the large activated platelets in the vasculature.

Also, it is known that lung function worsens during the exacerbation period. ${ }^{22}$ In our study, mean platelet volume was correlated with percent-of-predicted $\mathrm{FEV}_{1}$ but not significantly. Stability of the disease leads to lung function parameters in pre-exacerbation baseline conditions (data are not shown).

A limitation of our study is that the number of exacerbations was not assessed. Also, the cases in the study were relatively severe forms of exacerbation requiring hospitalization. Moreover, there was incomplete control of lung function.

\section{Conclusions}

In conclusion, mean platelet volume is an easily accessible low cost marker. The present study shows that a decrease of mean platelet volume occurs when COPD exacerbation inflammatory processes occur. Moreover, the decrease of mean platelet volume correlated with clinical and laboratory features that accompany COPD exacerbation. Thus, a decrease in mean platelet volume values may indicate exacerbations of COPD. Future studies should investigate whether mean platelet volume obtained as part of routine laboratory testing could identify patients at risk for an exacerbation event in subjects with COPD. Also, there is a need to explain the mechanism regulating platelet volume during COPD exacerbation through larger studies in the future.

\section{REFERENCES}

1. Global Initiative for Chronic Obstructive Lung Disease. Global strategy for the diagnosis, management, and prevention of chronic obstructive pulmonary disease. Updated 2015. http://www.goldcopd. org/uploads/users/files/GOLD_Report_2015_Feb18.pdf.

2. Seemungal TA, Hurst JR, and Wedzicha JA. Exacerbation rate, health status and mortality in COPD: a review of potential interventions. Int J Chron Obstruct Pulmon Dis 2009;4:203-223.

3. Koutsokera A, Stolz D, Loukides S, Kostikas K. Systemic biomarkers in exacerbations of COPD: the evolving clinical challenge. Chest 2012;141(2):396-405.

4. Weyrich AS, Lindemann S, Zimmerman GA. The evolving role of platelets in inflammation. J Thromb Haemost 2003;1(9):1897-1905.

5. Merolla M, Nardi MA, Hu L, Rockman CB, and Berger JS. Platelet size is an excellent surrogate for increased platelet activity. J Am Coll Cardiol 2011;57(14):E1600.

6. Bancroft AJ, Abel EW, Mclaren M, Belch JJ. Mean platelet volume is a useful parameter: a reproducible routine method using a modified Coulter thrombocytometer. Platelets 2000;11(7):379-387.

7. Dastjerdi MS, Emami T, Najafian A, Amini M. Mean platelet volume measurement, EDTA or citrate? Hematology 2006;11(5):317319.

8. Biljak VR, Pancirov D, Cepelak I, Popović-Grle S, Stjepanović G, Grubišić TŽ. Platelet count, mean platelet volume and smoking status in stable chronic obstructive pulmonary disease. Platelets. 2011; 22(6):466-470.

9. Steiropoulos P, Papanas N, Nena E, Xanthoudaki M, Goula T, Froudarakis $\mathrm{M}$, et al. Mean platelet volume and platelet distribution width in patients with chronic obstructive pulmonary disease: the role of comorbidities. Angiology 2013;64(7):535-539.

10. Ulasli SS, Ozyurek BA, Yilmaz EB, Ulubay G. Mean platelet volume as an inflammatory marker in acute exacerbation of chronic obstructive pulmonary disease. Pol Arch Med Wewn 2012;122(6): 284-290.

11. Wang RT, Li JY, Cao ZG, Li Y. Mean platelet volume is decreased during an acute exacerbation of chronic obstructive pulmonary disease. Respirology 2013;18(8):1244-1248.

12. Erden ES, Dokuyucu R, Demirköse M, Yengil E, Sefil F, Bilgiç HK, et al. Assessment of mean platelet volume in chronic obstructive pulmonary disease during stable period and acute exacerbation. J Clin Exp Invest 2013;4(4):483-487.

13. Bathoorn E, Liesker JJ, Postma DS, Koëter GH, van der Toorn M, van der Heide S, Kerstjens HA. Change in inflammation in outpatient COPD patients from stable phase to a subsequent exacerbation. Int J Chron Obstruct Pulmon Dis. 2009;4:101-109.

14. Wouters EF. Local and systemic inflammation in chronic obstructive pulmonary disease. Proc Am Thorac Soc 2005;2(1):26-33.

15. Wedzicha JA, Seemungal TA, MacCallum PK, Paul EA, Donaldson GC, Bhowmik A, et al. Acute exacerbations of chronic obstructive pulmonary disease are accompanied by elevations of plasma fibrinogen and serum IL-6 levels. Thromb Haemost 2000;84(2):210-215.

16. Hurst JR, Donaldson GC, Perera WR, Wilkinson TM, Bilello JA, Hagan GW, et al. Utility of plasma biomarkers at exacerbation of chronic obstructive pulmonary disease. Am J Respir Crit Care Med 2006;174(8):867-874. 


\section{Mean Platelet Volume in COPD Exacerbation}

17. Gan WQ, Man SF, Senthilselvan A, Sin DD. Association between chronic obstructive pulmonary disease and systemic inflammation: a systemic review and a meta-analysis. Thorax 2004;59(7):574-580

18. Barnes PJ, Celli BR. Systemic manifestations and comorbidities of COPD. Eur Respir J 2009;33(5):1165-1185.

19. Polosa R, Malerba M, Cacciola RR, Morjaria JB, Maugeri C, Prosperini $\mathrm{G}$, et al. Effect of acute exacerbations on circulating endothelial, clotting and fibrinolytic markers in COPD patients. Intern Emerg Med 2013;8(7):567-574.

20. Maclay JD, McAllister DA, Johnston S, Raftis J, McGuinnes C, Deans A, et al. Increased platelet activation in patients with stable and acute exacerbation of COPD. Thorax 2011;66(9):769-774.

21. Gasparyan AY, Ayvazyan L, Mikhailidis DP, Kitas GD. Mean platelet volume: a link between thrombosis and inflammation? Curr Pharm Des 2011;17(1):47-58.
22. Papi A, Bellettato CM, Braccioni F, Romagnoli M, Casolari P, Caramori G, et al. Infections and airway inflammation in chronic obstructive pulmonary disease severe exacerbations. Am J Respir Crit Care Med 2006;173(10):1114-1121.

23. Agapakis DI, Tsantilas D, Psarris P, Massa EV, Kotsaftis P, Tziomalos K, Hatzitolios AI. Coagulation and inflammation biomarkers may help predict the severity of community-acquired pneumonia. Respirology 2010;15(5):796-803.

24. Uysal P, Tuncel T, Olmez D, Babayigit A, Karaman O, Uzuner N. The role of mean platelet volume predicting acute exacerbations of cystic fibrosis in children. Ann Thorac Med 2011;6(4):227230.

25. Sun WX, Zhang JR, Cao ZG, Li Y, Wang RT. A decreased mean platelet volume is associated with stable and exacerbated asthma. Respiration 2014;88(1):31-37. 